\title{
Energy Circulation Methods for Surface Acoustic Wave Motor
}

\author{
Katsuhiko Asai, ${ }^{1}$ Minoru Kuribayashi Kurosawa, ${ }^{2}$ and Toshiro Higuchi ${ }^{3}$ \\ ${ }^{1}$ Advanced Technology Research Laboratories, Matsushita Electric Industrial Co., Ltd., Kyoto, 619-0237 Japan \\ ${ }^{2}$ Department of Advanced Applied Electronics, Tokyo Institute of Technology, Yokohama, 226-8502 Japan \\ ${ }^{3}$ Department of Precision Machinery Engineering, The University of Tokyo, Tokyo, 113-8656 Japan
}

\section{SUMMARY}

It is important to improve efficiency of surface acoustic wave motors in order to achieve their commercialization. In the present research, the energy of the surface wave, which has previously not been used effectively for driving the slider, but been consumed in sound-absorbing materials, is now circulated by two techniques, so that the surface wave is excited efficiently. Trial fabrication of surface acoustic wave motors using the energy circulation methods is carried out. It is confirmed that the traveling wave can actually be excited. In addition, the effectiveness of the energy circulation method is demonstrated by comparison of the driving performance with that in the absence of energy circulation methods. (C) 2003 Wiley Periodicals, Inc. Electron Comm Jpn Pt 3, 87(2): 10-19, 2004; Published online in Wiley InterScience (www.interscience. wiley.com). DOI 10.1002/ecjc.10132

Key words: surface acoustic wave; actuator; energy circulation; higher efficiency; MEMS.

\section{Introduction}

Surface acoustic wave motors are a type of actuators that are superior in terms of driving force, speed, driving precision, and energy density. Further, since the stator of the surface acoustic wave device and the silicon-based slider are fabricated by micromachine techniques cultivated in the MEMS field, the reliability and reproducibility of the device are excellent. However, the efficiency of the surface acoustic wave motors realized by research up to this time is essentially less than $1 \%$. It is therefore important for commercialization to improve the efficiency. The efficiency of the surface acoustic wave motors can be considered from two aspects. One is the efficiency of conversion from the vibration energy of Rayleigh waves to the output at the slider. The other is the efficiency of conversion of the input electric energy to the vibrating energy of Rayleigh waves used for driving the slider. The first item depends on the contact surface shape and the contact condition of the slider. The slider output can be improved by improving the contact surface area between the stator and the stator substrate and optimization of the preload $[1,2]$. The second item depends on the material and design of the stator substrate. In the surface acoustic wave motors studied to date, only a slight portion of the input energy is used for driving of the slider. The remaining energy is consumed in the absorber. In order to drive the slider efficiently, it is necessary to circulate this hitherto wasted energy [3].

A method of energy circulation for the surface acoustic wave motor has been reported by Tojo and colleagues [4]. In that report, a method for circulating the energy with two driving IDTs and four unidirectional electrodes on the stator substrate is presented. By providing input signals whose phase difference is $90^{\circ}$ to two driving IDTs, excitation of a traveling wave with a standing wave ratio of 1.6 is confirmed. However, no report has yet reported success in driving an energy circulation surface acoustic wave motor or discussed its design.

(C) 2003 Wiley Periodicals, Inc. 
In the present research, two new energy circulation methods are proposed for surface acoustic wave motors. Surface acoustic wave motors using these energy circulation methods are trial fabricated. Their effectiveness is presented relative to the case without energy circulation in terms of excitation efficiency and driving performance.

\section{Principle of Energy Circulation Surface Acoustic Wave Motor}

The surface acoustic wave motor is an actuator that derives its driving force through frictional forces from a Rayleigh wave, which is a type of surface acoustic wave. In the surface acoustic wave motors used in research to date, the Rayleigh wave is excited by one IDT. This scheme is called a single IDT type. A rough sketch of the surface acoustic wave motor of a single IDT type is shown in Fig. 1. In this scheme, the Rayleigh wave is excited by the energy that is input into both sides of the IDT. At both edges of the stator substrate, absorbers are attached to prevent reflection of the Rayleigh wave. The Rayleigh wave propagating in the direction opposite to the slider is directly absorbed by the absorber. The Rayleigh wave propagating toward the slider imparts a driving force to the slider at the contact surface and then is absorbed by the absorber. Hence, except for a portion of the energy used for driving the slider, all of the remaining energy is absorbed by the absorbers. Therefore, substantial power is required for driving a surface acoustic wave motor. In addition, in order to prevent breakdown of the substrate due to heating of the absorbers, burst driving needs to be used instead of continuous driving.

In order to handle this problem, it is necessary to make the excitation of the Rayleigh wave unidirectional and to circulate the vibrating energy of the Rayleigh wave transmitted through the contact surface with the slider. In the present research, two methods are proposed for energy circulation in the surface acoustic wave motor. One is an improvement of the energy circulation method originally proposed by Tojo's group mentioned above. The other uses

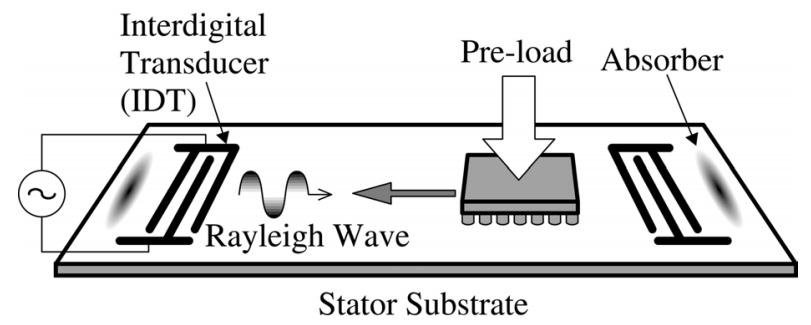

Fig. 1. Schema of the single IDT-type SAW motor. a combiner to circulate the energy. These methods are called the resonance type and the combiner type, respectively. In the following, both methods are explained briefly.

\subsection{Principle of the resonance-type energy circulation method}

The configuration of the resonance type is shown in Fig. 2. This type consists of two unidirectional IDTs installed at both ends of the stator substrate and a set of IDTs for driving. The two unidirectional IDTs are electrically connected. The directionality of the unidirectional IDTs is toward the center of the substrate. When an AC signal is applied to one of the driving IDTs, traveling waves are excited on both sides. The traveling wave arriving at the unidirectional IDT is converted to electrical signals and then reconverted to a traveling wave by another unidirectional IDT. Hence, the traveling wave is not reflected at the substrate edge but is circulated. If the reradiated wave is superposed on the original wave with the same phase, then the standing wave formed by superposition of the traveling waves in the two directions becomes resonant and its amplitude increases up to a certain value, corresponding to the loss in the circulation. This behavior is very similar to that in the ring-type ultrasonic motor. By applying sine wave and cosine wave AC signals to two driving IDTs placed with a spacing corresponding to one-quarter of the wavelength of the traveling wave, a traveling wave moving in one direction can be excited. Hence, a traveling wave can be excited without the energy consumption in the absorber that occurs in the case of the single IDT type. The sum of the energies input from the two IDTs is equal to the loss during circulation. However, there are various second-order effects of IDTs. Therefore, these effects must be taken into account in the design.

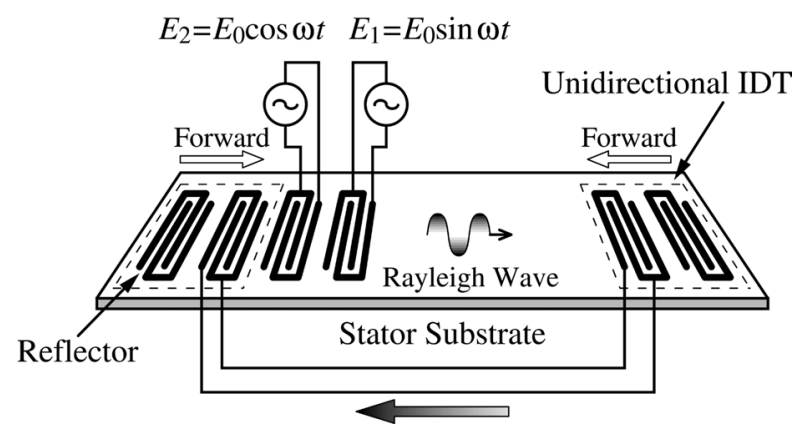

Fig. 2. Structure of the stator transducer using the resonance-type energy circulation method. 


\subsection{Principle of combiner-type energy circulation method}

The configuration of the combiner type is shown in Fig. 3. As seen, this method consists of two unidirectional IDTs at the ends of the stator substrate and a combiner. The output terminal of the combiner is connected to one of the unidirectional IDTs and the input terminal is connected to the other unidirectional IDT and to a high-frequency source. The directionality of the unidirectional IDTs is toward the center of the substrate as in the resonance type. The traveling wave propagating to the right on the stator substrate is converted to an electrical signal by the unidirectional IDT connected to the input terminal of the combiner. This energy $E_{2}$ and the energy $E_{p s}$ from the high-frequency source are combined to become $E_{1}\left(=E_{2}+\right.$ $E_{p s}$ ). By means of this energy, a traveling wave is excited once again by the unidirectional IDT connected to the output terminal of the combiner, so that the energy is circulated.

Within the combiner, the input energy from the power supply and the energy of the traveling wave are combined in an unbalanced manner. The efficiency is higher if the combining ratio $E_{2} / E_{p s}$ of the traveling wave energy to the input energy is larger. However, since

$$
E_{1}-E_{2} \leqq E_{p s}
$$

must be satisfied, the limit of the combining ratio is determined by the loss during circulation.

\subsection{Comparison between the resonance type and combiner type}

The energy circulation methods form a circulation path for the energy via the propagation path of Rayleigh wave and an electrical circuit. The resonance type combines

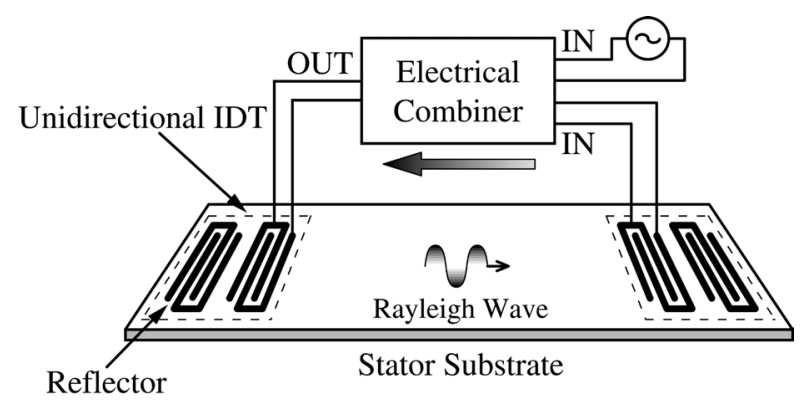

Fig. 3. Structure of the stator transducer using the combiner-type energy circulation method. the input energy from the electrical power supply and the circulating energy on the propagation path. The combiner type performs this function on the electrical circuit. They have the following advantages.

(a) Resonance type

- Fabrication is easier since no combiner is required.

- The driving direction can be switched by reversing the phase of the biphase signals.

- The efficiency can be enhanced to the maximum extent by improving the design.

(b) Combiner type

- Design of the stator substrate is easier than for the resonance type.

- Since no driving electrode such as is used in the resonator type is needed, it is easier to provide a running surface for the slider.

- A single phase power supply can be used for driving.

- Since the combining ratio is determined in the combiner, the effects of the motor conditions and external environment on the driving performance are small.

\section{Design of Energy Circulation Surface Acoustic Wave Motor}

By means of an equivalent circuit, surface acoustic wave motors using the energy circulation method are designed. As the equivalent circuit for the IDT, the circuit described by Smith and colleagues [5] is improved by Kojima and Shibayama [6]. The material for the stator substrate is $127.8^{\circ}$ rotation Y cut-X propagation $\mathrm{LiNbO}_{3}$. The driving frequency is $14.45 \mathrm{MHz}$. Figure 4 illustrates

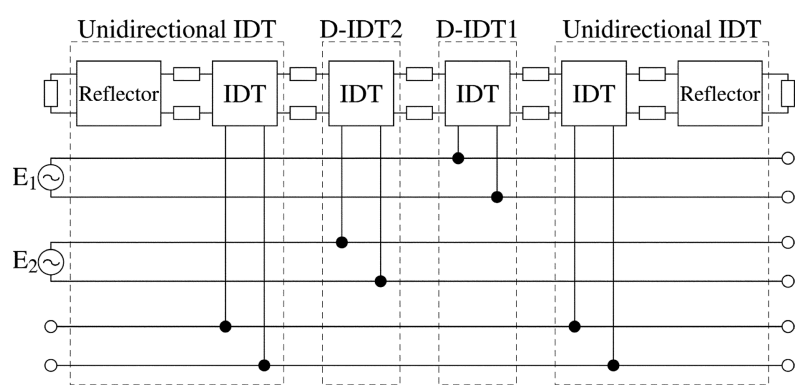

Fig. 4. Equivalent circuit of the stator transducer using the resonance-type energy circulation method. 
the eight-terminal pair equivalent circuit of the stator substrate for a resonant energy circulation surface acoustic wave motor. The equivalent circuit of the combiner-type energy circulating surface acoustic wave motor is shown in Fig. 5 with the slider removed. If the loss, phase variations, and effects of frequency are neglected, the combiner can be expressed by the four-terminal pair equivalent circuit shown in Fig. 6. Here, $n$ is the combining ratio. When energy $P$ is incident upon Input 1 and energy $n P$ upon Input 2 , energy $(n+1) P$ is extracted from Output 1 .

\subsection{Design of unidirectional IDT}

First, the design of the unidirectional IDTs placed on both ends of the stator substrate is described. In this research, unidirectional IDTs with the same design are used for both the resonance type and the combiner type. The unidirectional IDT consists of two IDTs. One is the IDT for electromechanical conversion (the conversion electrode) and the other is a reflector. The conversion IDT is designed in such a way that the conductance is maximal at the driving frequency, while the reflector is designed in such a way that the reflection is maximal at the driving frequency. By using the same designs for the two unidirectional IDTs on the ends of the substrate, their admittance values are made identical. However, if the spacing between the conversion IDT and the reflector is selected such that the conductance at the driving frequency is maximized, the susceptance is not zero. Hence, the matching condition cannot be satisfied if two unidirectional IDTs are connected electrically. Hence, the spacing between the conversion IDT and the reflector is adjusted so that the susceptance at the driving frequency becomes zero. As a result of the above design, the unidirectional IDTs are determined as given in Table 1. Also, the propagation path length between the conversion electrode and the reflector is $603 \mu \mathrm{m}$. The impedance of the unidirectional IDT at the driving frequency as derived from the equivalent circuit is $50 \Omega$ and the directivity is $43.6 \mathrm{~dB}$.

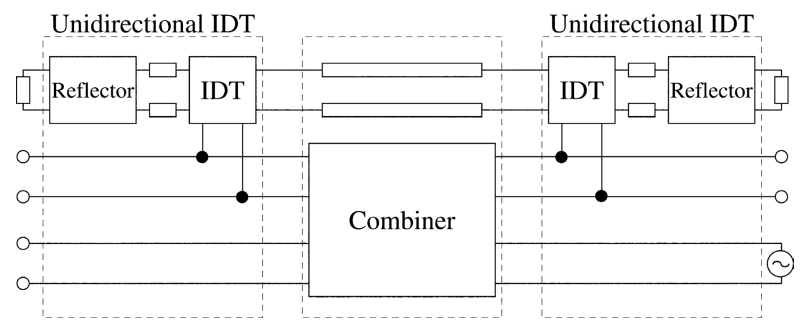

Fig. 5. Equivalent circuit of the stator transducer using the combiner-type energy circulation method.

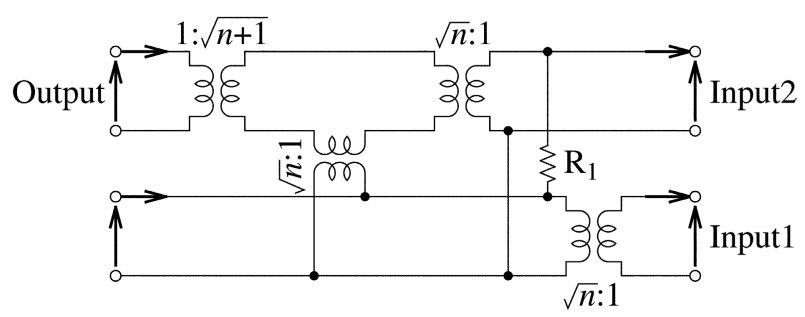

Fig. 6. Equivalent circuit of the electrical combiner.

\subsection{Design of resonance type}

In the resonance type, two driving IDTs are needed in addition to the unidirectional IDTs. The number of strip electrode pairs of driving IDTs is 10 and the size and positions of the electrodes are designed such that a traveling wave can be excited in the circulation path when signals with a phase difference of $90^{\circ}$ are applied. As a result, the period length of the driving IDTs is $272 \mu \mathrm{m}$, the electrode strip width is $68 \mu \mathrm{m}$, and the aperture size is $8 \mathrm{~mm}$. The propagation path length between two driving IDTs is 462 $\mu \mathrm{m}$. In this case, the directivity is about $14.6 \mathrm{~dB}$ when the two driving IDTs are considered as a biphase unidirectional IDT. However, the effect on the standing wave excited by one of the driving IDTs by the load connected to another driving IDT then becomes the minimum. Also, since 462 $\mu \mathrm{m}$ is approximately $(7 / 4) \lambda$, if a sine wave and a cosine wave are applied to the two driving IDTs, the traveling wave propagates toward the electrode to which the cosine wave is applied. Next, the distance between the driving IDT and the unidirectional IDT is designed in such a way that resonance is obtained at the driving frequency. As a result, the distance between D-IDT1 and the unidirectional IDT is $12.104 \mathrm{~mm}$ and that between D-IDT2 and the unidirectional IDT is $0.500 \mathrm{~mm}$. The numerical results of the admittance characteristics of the designed stator substrate are presented in Fig. 7. Here, the D-IDT1 and D-IDT2 curves indicate the numerical results at each driving IDT. The calculations are carried out under the assumption that the other IDT is open.

Table 1. Dimensions of the unidirectional IDT

\begin{tabular}{|c|c|c|}
\hline & Conversion IDT & Reflector \\
\hline Periodic length & $266 \mu \mathrm{m}$ & $274 \mu \mathrm{m}$ \\
\hline Electrode strip width & $66.5 \mu \mathrm{m}$ & $68.5 \mu \mathrm{m}$ \\
\hline Aperture size & $8 \mathrm{~mm}$ & $8 \mathrm{~mm}$ \\
\hline Strip electrode pairs & 21 & 34 \\
\hline
\end{tabular}


Here, D-IDT1 and D-IDT2 denote the driving IDTs located inside and outside in Fig. 2. As shown in Fig. 7, the driving IDT is at resonance at the driving frequency. By calculations based on the equivalent circuit, the power needed for excitation of the surface wave is estimated to be $15 \%$ of what is needed in the single IDT type when a cosine wave is applied to D-IDT1 and a sine wave to D-IDT2.

\subsection{Design of combiner type}

In the combiner method, a hybrid combiner using a toroidal core is developed. First, for the combining ratio, $n$ $=4$ is chosen with regard to ease of fabrication and loss of energy. The resistance $R_{1}=(n+1) \times 50=250 \Omega$ is chosen because the output impedance of the power supply and the impedance of the unidirectional IDT are $50 \Omega$. In the combiner method, energy circulation can be carried out efficiently if the sum of the phase difference between Input 2 and the Output in the combiner at the driving frequency and the phase difference between the unidirectional IDT connected to Output and that connected to Input 2 is made equal to an integer multiple of $2 \pi$. The propagation length between the two unidirectional IDT's is chosen as 18,560 $\mu \mathrm{m}$ with the phase variation in the combiner taken into account. Figure 8 shows the calculation results of the transfer characteristic in the $50-\Omega$ system of the designed stator substrate. One of the unidirectional IDTs is on the input side and the other is on the load side. From Fig. 8, it is found that the loss takes the minimum value of $1.3 \mathrm{~dB}$ at a driving frequency of $14.45 \mathrm{MHz}$. When $E_{2} / E_{1}=-1.3 \mathrm{~dB}$ and $E_{p s} / E_{2}$ $=0.25$ are substituted into Eq. (1), the equation is satisfied because $0.16 \leq 0.25$. If the loss in the combiner is neglected,

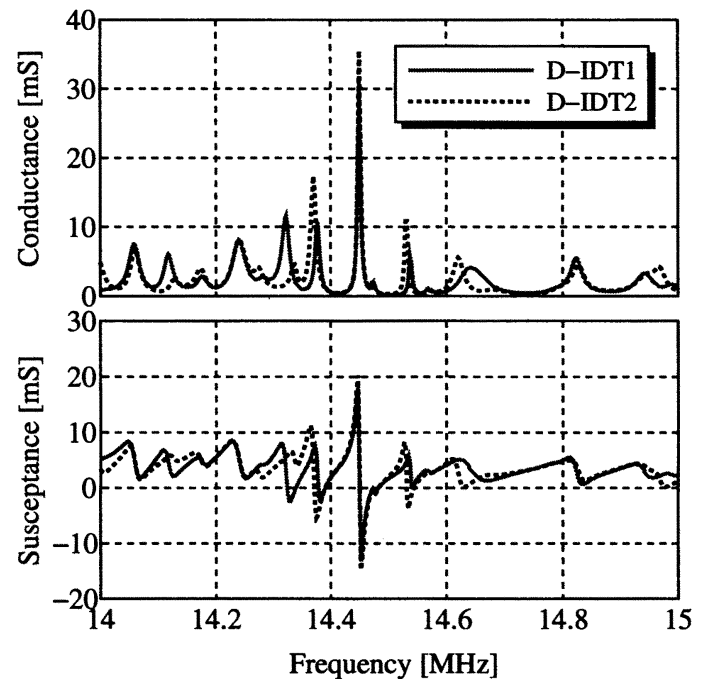

Fig. 7. Calculated admittance of driving IDT.

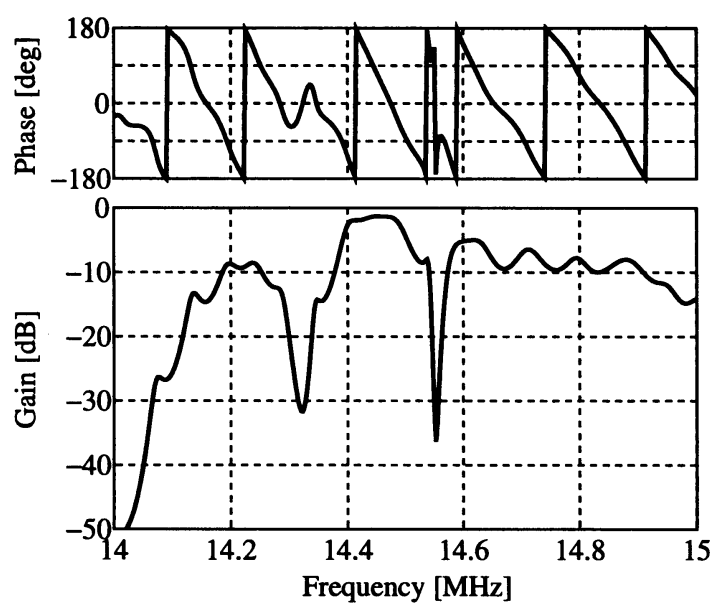

Fig. 8. Calculated transfer function.

then $E_{p s}$ is $20 \%$ of $E_{1}$ in the stator substrate without the slider. Further, since the excitation of the surface wave is carried out by a unidirectional IDT, the power needed for excitation of the surface wave is estimated to be $10 \%$ of that needed in the single IDT type.

\section{Characteristics of Stator Substrate}

\subsection{Resonance type}

Based on the design results of the resonance-type energy circulation method, a stator substrate was fabricated. Figure 9 shows a photograph of the fabricated stator substrate. The measured admittance characteristic of the stator substrate is shown in Fig. 10. The results agree well with

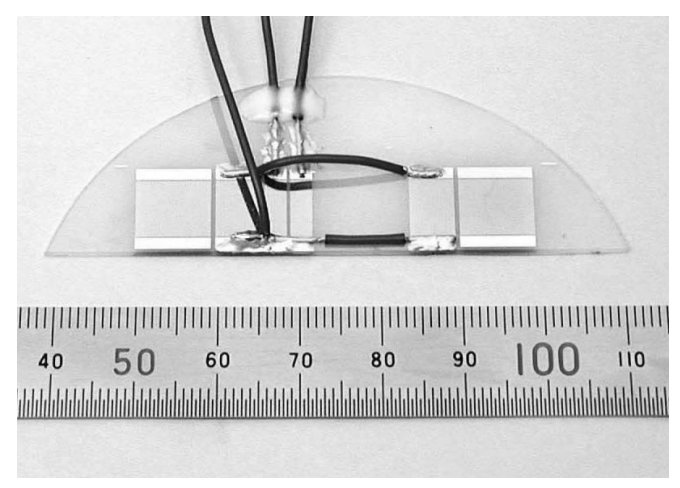

Fig. 9. Stator transducer for SAW motor using the resonance-type energy circulation method. 


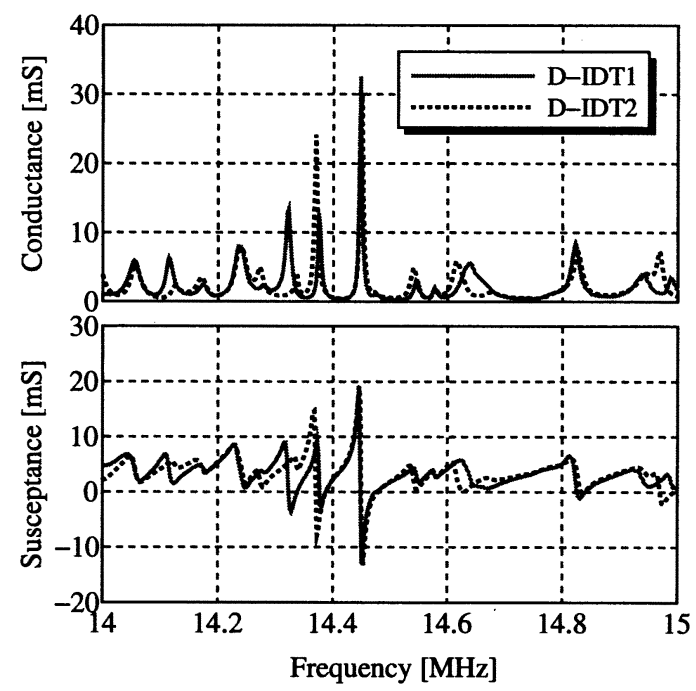

Fig. 10. Experimental result of admittance of driving IDT.

the calculation results in Fig. 7. Table 2 lists the experimentally and numerically determined $Q$ values at a resonant frequency of $14.450 \mathrm{MHz}$. As shown in this table, the results essentially agree with the computed values, although there is a slight deviation in the resonant frequency.

Next, excitation of a traveling wave is attempted by applying signals to the two driving IDTs. Figure 11 shows the vibration distribution measured by a laser interferometer. The positive direction in this figure is for the traveling wave progressing from the driving IDT to the unidirectional IDT on the far side. The total of the input powers is $0.4 \mathrm{~W}$. The voltages applied to each of the driving IDTs are shown in Table 3. The reason why the input voltages to D-IDT1 and D-IDT2 are different is that the standing wave amplitudes excited by the IDTs are made equal. The difference in input voltage between the forward and reverse directions is attributed to the effect between the two driving IDTs, which is not completely eliminated. From Fig. 11, it is confirmed that traveling waves can be excited in both directions, although a slight amount of standing wave components is present. Thus, the standing wave ratio, the ratio

Table 2. $Q$ value at driving frequency

\begin{tabular}{lcc}
\hline & $\begin{array}{c}\text { Resonance } \\
\text { frequency }\end{array}$ & $Q$ value \\
\hline D-IDT1 (computed) & $14.450 \mathrm{MHz}$ & 2525 \\
D-IDT1 (measured) & $14.445 \mathrm{MHz}$ & 2789 \\
D-IDT2 (computed) & $14.450 \mathrm{MHz}$ & 2525 \\
D-IDT2 (measured) & $14.447 \mathrm{MHz}$ & 2421 \\
\hline
\end{tabular}

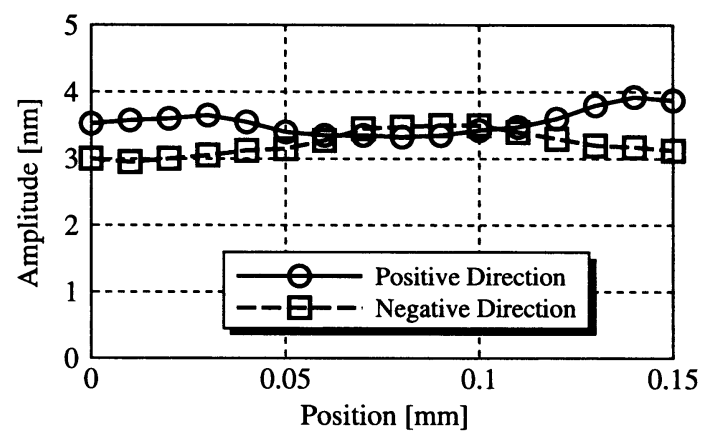

Fig. 11. Vibration distribution using the resonance-type energy circulation method.

of the maximum amplitude to the minimum amplitude, is 1.2 in both directions.

\subsection{Combiner type}

Based on the design results for the combiner-type energy circulation method, a stator substrate was fabricated. Figure 12 shows a photograph of the fabricated stator substrate. The measured transfer function in the 50- $\Omega$ system on the stator substrate is shown in Fig. 13. Like the calculation results in Fig. 8, one of the unidirectional IDTs is on the input side and the other is on the load side. The loss is smallest in the transfer characteristic at $14.42 \mathrm{MHz}$ and is $1.3 \mathrm{~dB}$. The minimum value of the loss is close to the calculation result. However, the frequency is different from the computed value. This is attributed to the fact that the admittance of the unidirectional IDT is different from the computed value. Therefore, for efficient energy circulation, the phase must be adjusted. By some adjustment of the combiner and by interchange of the ground side of one of the unidirectional IDTs, the efficient energy circulation was attained at $14.40 \mathrm{MHz}$ with a loss of $1.9 \mathrm{~dB}$. In the subsequent experiment, the driving frequency was $14.40 \mathrm{MHz}$.

Figure 14 shows the measured vibration distribution when a traveling wave is excited with an input power of 0.7 $\mathrm{W}$. The standing wave ratio obtained is 1.4 , somewhat

Table 3. Input voltage in measurement of vibration amplitude

\begin{tabular}{llc}
\hline & & Input voltage \\
\hline Forward direction & D-IDT1 & $3.53 \mathrm{~V}_{\text {peak }}$ \\
& D-IDT2 & $4.36 \mathrm{~V}_{\text {peak }}$ \\
Reverse direction & D-IDT1 & $2.10 \mathrm{~V}_{\text {peak }}$ \\
& D-IDT2 & $4.17 \mathrm{~V}_{\text {peak }}$ \\
\hline
\end{tabular}




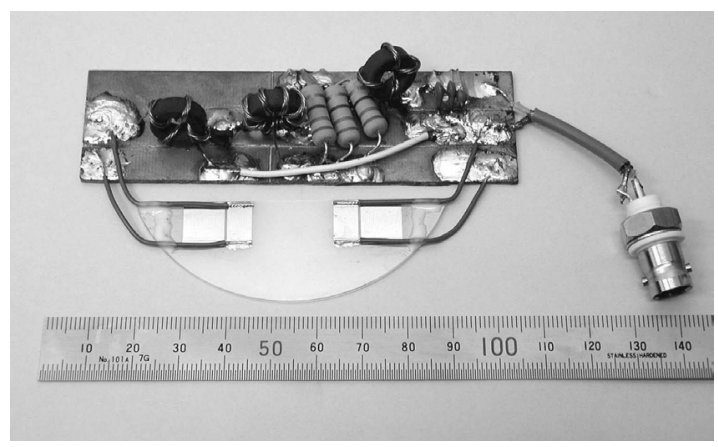

Fig. 12. Stator transducer and electrical combiner for SAW motor using the combiner-type energy circulation method.

poorer than that in the resonator method. It is confirmed that a traveling wave is excited.

\subsection{Comparison of power needed for excitation of traveling wave}

It is now confirmed that a traveling wave can be excited by either the resonance type or the combiner type of energy circulation method. Hence, the difference between the injected power and the driving voltage is measured when the amplitude of the excited traveling wave is fixed. The amplitude is taken to be that of the traveling wave corresponding to the propagating energy. When the vibration distributions shown in Figs. 11 and 14 are obtained, information on the standing wave ratio, maximum amplitude, and minimum amplitude can be obtained. By means of the minimum amplitude, the maximum amplitude is normalized so that the result is $x$ if the standing wave ratio

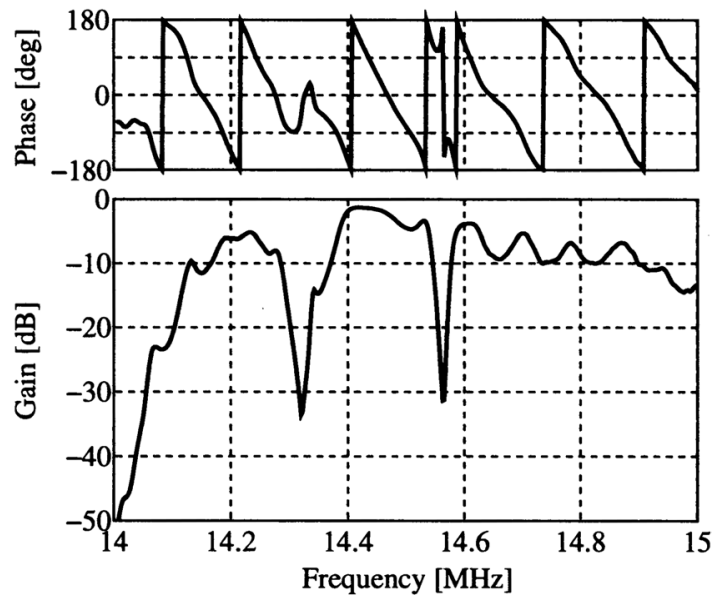

Fig. 13. Experimental result of transfer function.

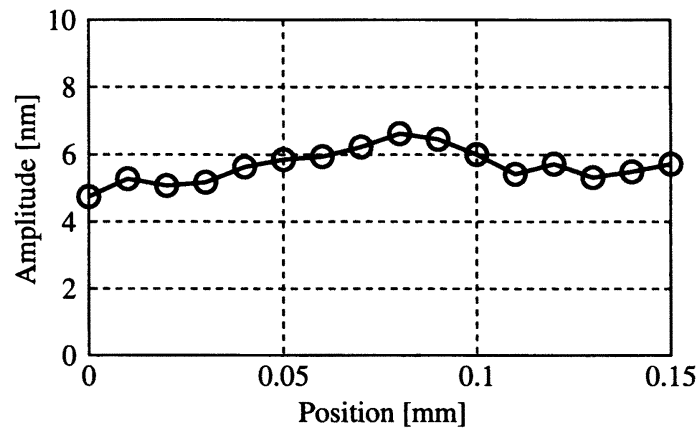

Fig. 14. Vibration distribution using the combiner-type energy circulation method.

is $x$. On the other hand, from the point of view of the transmitted wave in the propagation direction and the reflected wave propagating in the opposite direction, the sum of the amplitudes of the transmitted wave and the reflected wave is the maximum amplitude and the difference is the minimum amplitude. Hence, the amplitude of the transmitted wave is $(x+1) / 2$ and that of the reflected wave is $(x-$ $1) / 2$. The energy carried by each wave is proportional to the square of the amplitude. Hence, the amplitude of the traveling wave corresponding to the propagation energy, expressed as the difference of the energy by the transmitted wave and that of the reflected wave, is $\sqrt{x}$. If the standing wave ratio is small, the amplitude of the traveling wave is almost equal to the average of the maximum amplitude and the minimum amplitude. Hence, as the traveling wave amplitude in the case of energy circulation method, we use the value measured at the location of the average of the maximum amplitude and the minimum amplitude.

Figure 15 shows the measured relationship between the injected power and the traveling wave amplitude. In this figure, "Single IDT type" indicates the results given by the

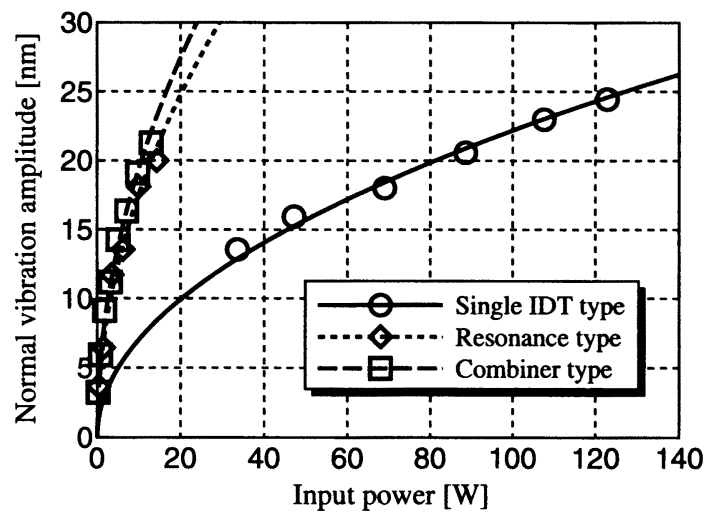

Fig. 15. Normal vibration amplitude versus input power. 


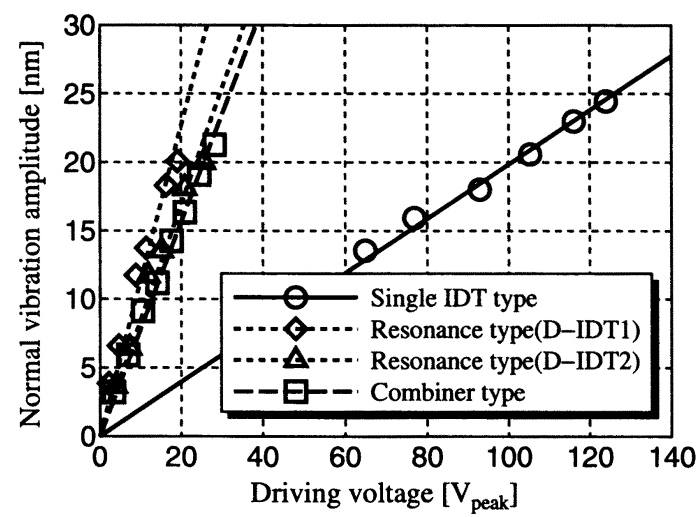

Fig. 16. Normal vibration amplitude versus driving voltage.

IDT, identical to the conversion IDT in the designed unidirectional IDT, with a number of pairs $N=21$, a period length $l=266 \mu \mathrm{m}$, a metallization ratio $\eta=0.5$, and an aperture size $w=8 \mathrm{~mm}$. In the resonance type, the results for forward propagation are presented. From Fig. 15, it is found that 14 $\mathrm{W}$ is needed in the resonance type and $11 \mathrm{~W}$ in the combiner type to obtain a traveling wave amplitude of $20 \mathrm{~nm}$. On the other hand, $81 \mathrm{~W}$ of power is needed in the single IDT type. Hence, it is confirmed that the power needed for excitation of the traveling wave is reduced to 17 and $14 \%$, respectively. These values are somewhat larger than the estimated values of 15 and $10 \%$. Nevertheless, the expected results are realized.

Figure 16 shows the measured relationship between the driving voltage and the traveling wave amplitude. From this figure, it is found that the driving voltage is also reduced to about $1 / 4$. The driving voltage comparison is not straightforward because the impedance values of the driving IDT are different in the different types.

\section{Driving Experiment}

With a fabricated energy circulating-type surface acoustic wave motor, a driving experiment was conducted for the slider. The configuration of the experimental setup is shown in Fig. 17. A silicon slider with dimensions of $4 \times$ $4 \times 0.3 \mathrm{~mm}^{3}$ was used. Cylindrical projections with a diameter of $20 \mu \mathrm{m}$ were installed every $30 \mu \mathrm{m}$ on the contact surface with the stator substrate. On the upper surface of the silicon slider, a permanent magnet was installed. By placing the stator substrate on top of a metal plate, preload was applied by means of a magnetic force. A weight was hung from a string attached to the slider via a pulley. By measuring the mass of the weight when the slider

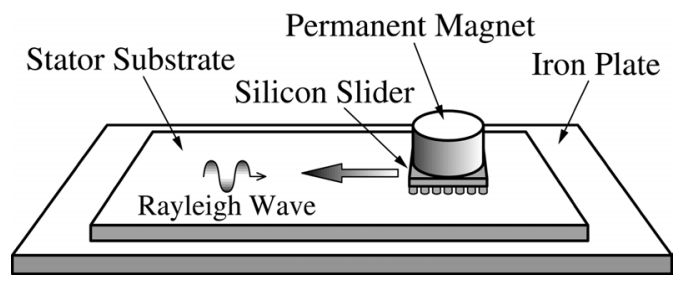

Fig. 17. Experimental setup.

was removed from the stator substrate, the preload was measured. As a result, the preload consisting of the mass of the slider and the magnetic force was $0.86 \mathrm{~N}$. The mass $m$ of the entire slider including the permanent magnet was $1.32 \mathrm{~g}$.

The speed variation of the slider when a driving voltage was applied to the energy circulation surface acoustic wave motor was measured with a laser Doppler vibration meter. Based on the measured transient response, the equations

$$
v=v_{0}\left(1-e^{-\frac{t}{\tau}}\right), \quad \tau=m \frac{v_{0}}{F_{0}}
$$

were fitted to obtain the steady speed $v_{0}$ and the maximum driving force $F_{0}$. The results are shown in Figs. 18 and 19. Also, from the relationship of $P_{\max }=v_{0} F_{0} / 4$, the maximum output $P_{\text {max }}$ was obtained; it is plotted in Fig. 20. As shown in these results, it is confirmed that a slider can be driven by a surface acoustic wave motor using the energy circulation method. The driving performance is similar in the single IDT type, resonance type, and combiner type at the

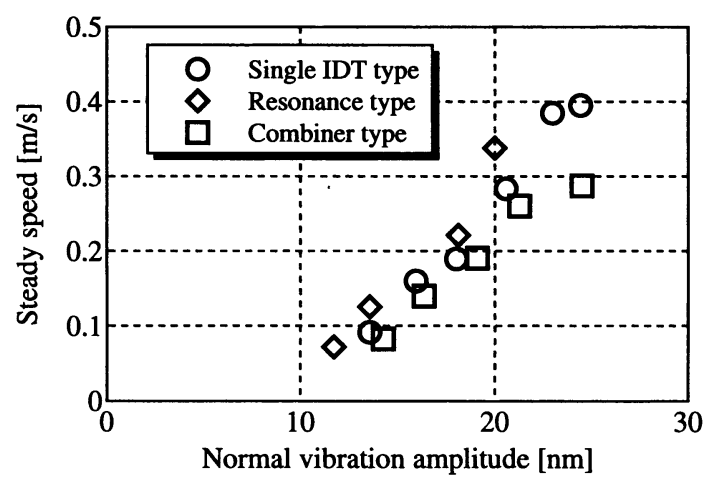

Fig. 18. Steady speed versus normal vibration amplitude. 


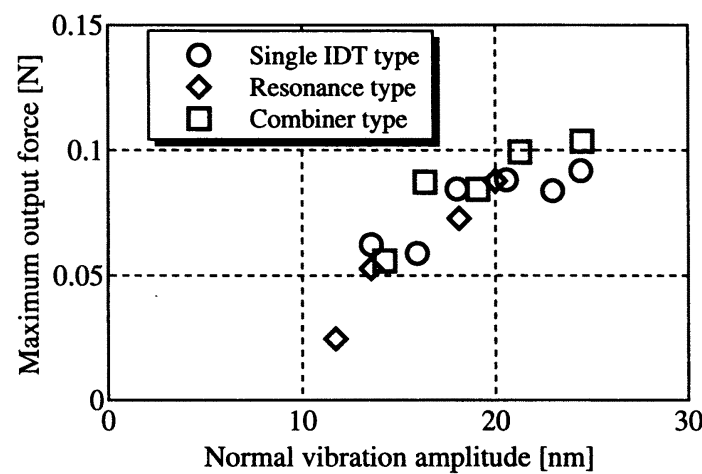

Fig. 19. Maximum output force versus normal vibration amplitude.

same surface wave amplitude. Thus, it is confirmed that the driving efficiency can be improved over the single IDT type if the energy circulation method of either the resonance type or the combiner type is used. However, the efficiency value obtained in the present driving experiment is about $0.06 \%$ even in the combiner type, because the conversion efficiency of the vibration energy of the Rayleigh wave to the slider output is low as a result of the smallness of the preload applied to the slider.

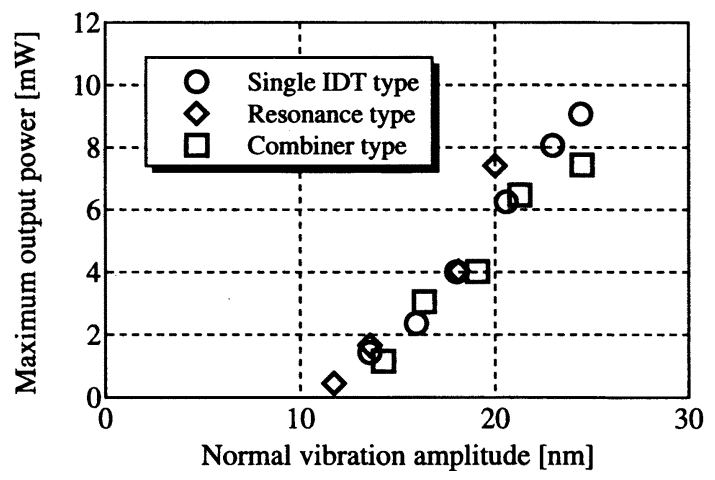

Fig. 20. Maximum output power versus normal vibration amplitude.

\section{Conclusions}

In this paper, two methods, the resonant type and the combiner type, are proposed for energy circulation in a surface acoustic wave motor. Surface acoustic wave motors based on these techniques were fabricated and tested. It was confirmed that a traveling wave can be excited in both methods. The power needed for excitation of the traveling wave is reduced to 17 and $14 \%$ in comparison to the single IDT type without energy circulation. Further, if the traveling wave amplitudes are kept the same, a driving performance almost identical to that in the single IDT type can be obtained experimentally in both methods. Hence, it is confirmed that the driving efficiency can be improved over that in the single IDT type by means of the two proposed energy circulation methods. However, in order to improve the driving performance of the surface acoustic wave motor and to increase its efficiency, the preload must be increased. The effects occurring under such conditions will be studied in the future.

\section{REFERENCES}

1. Kurosawa M, Takahashi M, Higuchi T. Optimum pre-load of surface acoustic wave motor. 1996 Proc IEEE Ultrasonics Symp, p 369-372, San Antonio.

2. Kurosawa M, Chiba M, Higuchi T. Multi contact points slider for a surface acoustic wave motor. Trans IEE Japan 1997;117-E:430-431.

3. Kuribayashi M, Ueha S, Mori E. Study of ultrasonic linear motor (5). Proc Meeting of Acoustic Society of Japan, p 585-586, 1985. (in Japanese)

4. Tojo K, Kurosawa M, Higuchi T. Circulated energy surface acoustic wave motor. Proc 10th Symposium on Electromagnetics and Dynamics, p 505-508, 1998. (in Japanese)

5. Smith WR, Gerard HM, Collins JH, Reeder TM, Shaw HJ. Analysis of interdigital surface wave transducers by use of an equivalent circuit model. IEEE Trans Microwave Theory Tech 1969;17:856864.

6. Kojima T, Shibayama K. An analysis of an equivalent circuit model for an interdigital surface-acousticwave transducer. Jpn J Appl Phys Suppl 1988;27: 163-165. 


\section{AUTHORS (from left to right)}
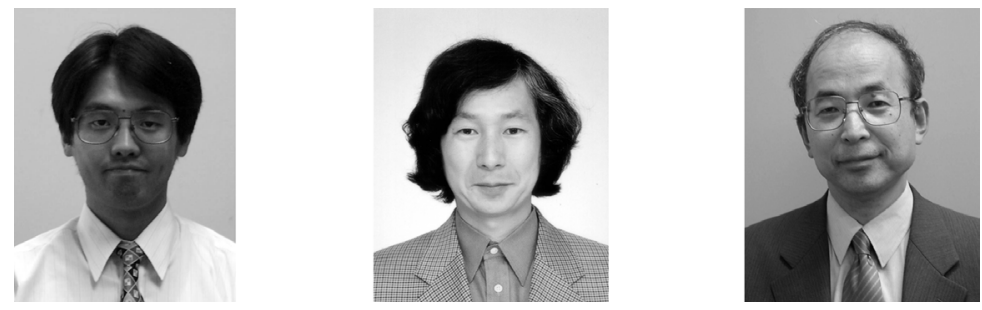

Katsuhiko Asai (member) received his B.S. and M.S. degrees in mechanical engineering from Tokyo Institute of Technology in 1992 and 1994 and joined Matsushita Electric Industrial Co. He has been engaged in research and development of micro-actuators. He is a member of the Japan Society of Mechanical Engineers.

Minoru Kuribayashi Kurosawa (member) received his B.E. degree in electrical and electronic engineering and M.E. and D.Eng. degrees from Tokyo Institute of Technology in 1982, 1984, and 1990. In 1984, he became a research associate in the Precision and Intelligence Laboratory of that Institute. He moved to the Department of Precision Machinery Engineering, Graduate School of Engineering, The University of Tokyo, in 1992 as an associate professor. Since 1999, he has been an associate professor in the Department of Advanced Applied Electronics, Interdisciplinary Graduate School of Engineering, Tokyo Institute of Technology. His current research interests include ultrasonic motors, micro-actuators, PZT thin films, SAW actuators, and single-bit digital signal processing and its application to control system. He is a member of the Acoustic Society of Japan, IEEE, the Institute of Electrical Engineers of Japan, and the Japan Society for Precision Engineering.

Toshiro Higuchi received his B.S., M.S., and Ph.D. degrees in precision machinery engineering from the University of Tokyo in 1972, 1974, and 1977. After serving as a lecturer and associate professor, he has been a professor in the Department of Precision Machinery Engineering, University of Tokyo, since 1991. He was the leader of the Higuchi Ultimate Mechatronics Project, Kanagawa Academy of Science and Technology, from 1992 to 1997. His research interests include mechatronics, magnetic bearings, electrostatic actuators, MEMS, robotics, and manufacturing. 